Check for updates

Cite this: RSC Adv., 2019, 9, 25225

Received 8th July 2019

Accepted 7th August 2019

DOI: 10.1039/c9ra05196a

rsc.li/rsc-advances

\section{Relation between the particle size and release characteristics of aromatic melamine microcapsules in functional textile applications}

\begin{abstract}
Hongbin Zhao, (D) a Xuening Fei, (DD *ab Lingyun Cao, ${ }^{b}$ Baolian Zhang ${ }^{c}$ and Xin $\mathrm{Liu}^{\mathrm{d}}$
The relation between the particle size and release characteristics of aromatic microcapsules with a melamine resin shell in functional textile applications have been investigated. Firstly, the microcapsules are characterized based on their Fourier transform infrared spectra, encapsulation efficiencies, particle size distributions, optical images, and scanning electron microscopy images. The impregnation performances of the microcapsules have been initially evaluated using image analysis. Subsequently, the impregnation efficiency and broken release characteristics are semi-quantitatively analyzed using solidphase microextraction-gas chromatography-mass spectrometry. The analysis results show that the highest impregnation efficiency and broken release intensity could be observed when the microcapsule size was similar to the fiber diameter $(25-30 \mu \mathrm{m})$. Eventually, the sustained release of the microcapsules over a period of $2400 \mathrm{~h}$ was evaluated using the weighing calculation method, and the trends were studied using the Peppas model. It was found that the microcapsule release rate slowly and continuously decreased with time, and the release rates significantly increased with the decrease in microcapsule particle size. Thus, it could be concluded that the large microcapsules exhibited better leak tightness than the small microcapsules, whereas the small microcapsules exhibited faster sustained release rates.
\end{abstract}

\section{Introduction}

Microencapsulation, as a protective technique designed to seal active ingredients within micro-scale particles, is widely considered to be an excellent delivery method for functional materials. Therefore, it is applied in several fields, including perfumes/fragrances, ${ }^{\mathbf{1 , 2}}$ antimicrobial agents, ${ }^{3}$ medicine, ${ }^{\mathbf{4}, \mathbf{5}}$ pigments, ${ }^{6,7}$ phase-change materials, ${ }^{8,9}$ self-healing materials, ${ }^{10,11}$ flame retardants, ${ }^{12}$ damage sensing, ${ }^{13}$ and lubricants. ${ }^{14}$ The release and delivery properties are the most critical functions of the microcapsules in several applications, and these characteristics have attracted extensive attention, especially in the health care and cleaning product industries. Various methods, including thermal gravimetric analysis, ${ }^{15}$ electronic nose, ${ }^{2}$ fluorescence microscopy, ${ }^{16}$ gas chromatography with flame ionization detection (GC-FID), ${ }^{1}$ high-performance liquid chromatography, ${ }^{17}$ Fourier transform infrared (FTIR) spectroscopy, ${ }^{18}$ and mathematical fitting, ${ }^{19-21}$ have been applied to study the release and delivery properties of the microcapsules.

${ }^{a}$ School of Chemical Engineering and Technology, Tianjin University, Tianjin 300350, China. E-mail: xueningfei@126.com

${ }^{b}$ School of Science, Tianjin Chengjian University, Tianjin 300384, China

${ }^{c}$ School of Materials Science and Engineering, Tianjin Chengjian University, Tianjin 300384, China

${ }^{d}$ Tianjin AnYing Bioengineering Technology Co., Ltd., Tianjin 300384, China
The particle size significantly affects the properties of microcapsules. Therefore, the control of microcapsule size has been extensively investigated in previous studies. ${ }^{15,22}$ However, there are still some problems which need to be clarified in the relationship between particle size and release behaviors of microcapsule.

In this study, we prepared aromatic melamine microcapsules with different particle sizes based on our previously developed method ${ }^{22}$ and characterized the microcapsules based on their FTIR spectra, encapsulation efficiencies $(E \%)$, particle size distributions, optical images, and scanning electron microscopy (SEM) images. Further, cotton fabrics were finished with prepared microcapsules, and their impregnation performances were initially investigated using image analysis. The impregnation efficiency and broken release characteristics of the microcapsules under the external force were semiquantitatively analyzed using solid-phase microextraction gas chromatography-mass spectrometry (SPME-GC-MS). Finally, the sustained release behavior of the microcapsules over a period of $2400 \mathrm{~h}$ were evaluated using the weighing calculation method. The sustained release data were fitted using a mathematical model, and the effects of particle size were discussed.

\section{Material and methods}

\subsection{Materials}

Methanol-modified melamine-formaldehyde (MMF) pre-polymer, the shell material, was purchased from Honisite Chemical Trade 
Co., Ltd. (Tianjin, China). The core material essence oil (Rose® 7289) was supplied by Double Horse Flavor and Fragrance Trade Co., Ltd. (Tianjin, China). The dispersant was styrene maleic anhydride (SMA) copolymer (Scripset巴 ${ }^{\circledR}$ 520, Hercules, USA). Acetic acid $(50 \mathrm{wt} \%)$ and sodium hydroxide $(\mathrm{NaOH})$ were purchased as analytical reagents (Tianjin Kermel Chemical Reagent Development Center, China). The properties of the fabric substrate used in this study are presented in Table 1.

\subsection{Preparation of MMF microcapsules}

Microcapsules containing essence oil were prepared via in situ polymerization using MMF as the shell material. The essence oil (20 g), 10 wt\% SMA aqueous solution (20 g) and deionized (DI) water (45 g) were mixed and mechanically emulsified. The prepared emulsion was poured into four-neck round-bottomed flask equipped with the reflux condenser and PTFE anchor stirrer, and the MMF pre-polymer (8.5 g) dissolved in DI water $(6.5 \mathrm{~g})$ was added. The emulsion $\mathrm{pH}$ was adjusted to 4 by $50 \mathrm{wt} \%$ acetic acid aqueous solution (AS), and the temperature was slowly increased to $75{ }^{\circ} \mathrm{C}$. After $1.5 \mathrm{~h}$ polymerization, the solution $\mathrm{pH}$ was adjusted to 7 with $30 \mathrm{wt} \% \mathrm{NaOH}$ AS, and obtained the microcapsule samples. Detailed particle size control methods reference to our previous study. ${ }^{22}$

\subsection{Impregnation of the microcapsules on fabric substrates}

The prepared aromatic microcapsule emulsion (2 g) and DI water $(198 \mathrm{~g})$ were mixed in a $500 \mathrm{~mL}$ glass beaker to produce a fabric finishing agent (1.0 wt\%). The fabric substrate (Table 1) was subsequently impregnated in a solution for $10 \mathrm{~min}$ at $25{ }^{\circ} \mathrm{C}$. After removing the fabric from the solution, the fabric was completely dried in an air-circulating oven at $50{ }^{\circ} \mathrm{C}$.

\subsection{Characterization}

2.4.1 FTIR. The microcapsule suspension was filtered, washed for times using DI water, and dried for $12 \mathrm{~h}$ in a vacuum oven at $50{ }^{\circ} \mathrm{C}$ to obtain a microcapsule sample without any nonencapsulated essence oil and free water. All the samples were analyzed using a Thermo Nicolet 380 FTIR spectrometer (Thermo Fisher Scientific) using the KBr pellet method (transmittance mode, range $=400-4000 \mathrm{~cm}^{-1}$, resolution $4 \mathrm{~cm}^{-1}$ ).

2.4.2 Microcapsule particle size distribution. The particle size distributions of the untreated microcapsule samples were measured using a laser particle size distribution analyzer (LSPOPIII, Zhuhai OMEC Instrument Co., Ltd., China). The average particle size was represented to have a medium diameter $D_{50}$, and the size distribution was characterized using the particle size distribution coefficient $P$, as denoted using eqn (1).

Table 1 Properties of the fabric substrate

\begin{tabular}{|c|c|c|c|c|c|}
\hline Style & Weave & Weight $\left(\mathrm{g} \mathrm{m}^{-2}\right)$ & $\begin{array}{l}\text { Thickness } \\
(\mathrm{mm})\end{array}$ & Width (mm) & Length $(\mathrm{mm})$ \\
\hline Cotton & Plain & 156 & 0.4 & 6 & 18 \\
\hline
\end{tabular}

$$
P=\frac{D_{(4,3)}}{D_{(3,2)}}
$$

where $D_{(4,3)}$ denotes the volume mean diameter and $D_{(3,2)}$ denotes the area average diameter.

2.4.3 Optical microscopy. A drop of the prepared microcapsule suspension was placed on a slide and dried in an aircirculating oven at $50{ }^{\circ} \mathrm{C}$ for $5 \mathrm{~min}$. The obtained microcapsule samples and impregnated fabric samples (see Section 2.3) were observed via optical microscopy (OM) (B203 optical microscope, Chongqing Optec Instrument Co., Ltd., China).

2.4.4 SEM. The diluted microcapsule samples were dropped onto silicon plates and dried in an air-circulating oven at $50{ }^{\circ} \mathrm{C}$ for $24 \mathrm{~h}$. Further, the samples were sputter-coated with a conductive Au coating at $30 \mathrm{~mA}$ for $60 \mathrm{~s}$. The microstructures of the sample were then observed via SEM (JSM-7800F, JEOL Co., Ltd., Japan).

2.4.5 Evaluation of $\boldsymbol{E} \%$ and sustained release performance. The core material percentages $\left(M_{\text {core }} \%\right)$ and encapsulation efficiencies $(E \%)$ of prepared microcapsules were determined using a weighing calculation method. An analytical balance was used to determine the mass of an empty evaporating Petri dish $\left(M_{0}\right)$. A small amount of the prepared microcapsule sample was quickly poured into the dish to form a semi-transparent film, and its mass $\left(M_{1}\right)$ was recorded. The sample in the dish was dried in an air-circulating oven at $50{ }^{\circ} \mathrm{C}$ for $24 \mathrm{~h}$ to eliminate all the free water and the non-encapsulated core material; subsequently, the mass $\left(M_{2}\right)$ was recorded. The sample was further dried at $25{ }^{\circ} \mathrm{C}$ to simulate the dynamic release conditions under ambient temperature. The air flow rate $\left(V_{\mathrm{X}}\right)$ at 10 points around the samples were measured and found that they were basically the same $\left(V_{\mathrm{X}} \approx 0.1 \mathrm{~m} \mathrm{~s}^{-1}\right)$, thus the average air flow rate of the shaded area shown in Fig. 1 could considered to be $0.1 \mathrm{~m} \mathrm{~s}^{-1}$. The Petri dish was weighed at regular intervals $\left(M_{\mathrm{n}}\right)$. The essence oil mass $\left(M_{\text {core }}\right)$, percentage $\left(M_{\text {core }} \%\right), E \%$, and the percentage of remaining essence oil during sustained release process $(W \%)$ of each sample were calculated using eqn (2)-(5), respectively.

$$
\begin{gathered}
M_{\text {core }}=\left(M_{1}-M_{0}\right)(\alpha+\beta)-\left(M_{1}-M_{2}\right) \\
M_{\text {core }} \%=\frac{M_{\text {core }}}{M_{2}-M_{0}} \times 100 \%
\end{gathered}
$$
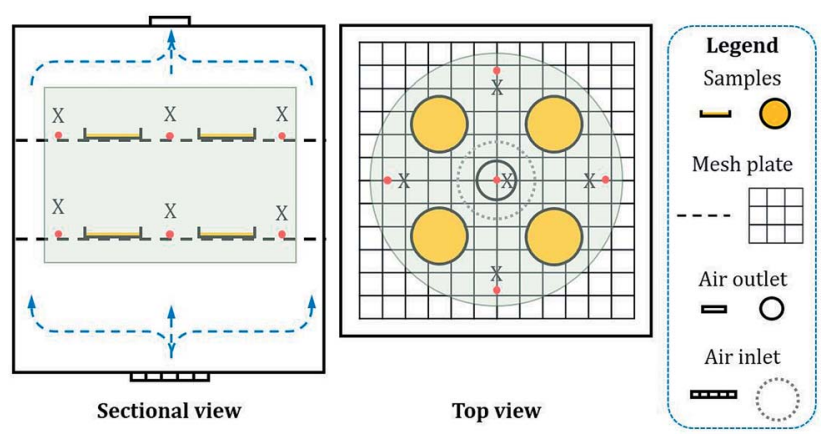

Fig. 1 Schematic diagram of the sustained release behavior test. 


$$
\begin{gathered}
E \%=1-\frac{\left(M_{1}-M_{2}\right)-\beta\left(M_{1}-M_{0}\right)}{\alpha\left(M_{1}-M_{0}\right)} \times 100 \% \\
W \%=\frac{M_{\text {core }}-\left(M_{2}-M_{\mathrm{n}}\right)}{M_{\text {core }}} \times 100 \%
\end{gathered}
$$

Here, $\alpha=0.20$ represents the mass fraction of the core material in the microcapsule sample, and $\beta \approx 0.71$ is the mass fraction of the volatile components (except the core material) in the sample.

2.4.6 Evaluation of the released aroma. The release properties of the microcapsule-treated fabrics were qualitatively and quantitatively analyzed by SPME-GC-MS. The finished fabric samples was placed in $4 \mathrm{~mL}$ vials and treated using the following hitting process. The vials were hit five times with a glass $\operatorname{rod}(\phi=5 \mathrm{~mm}$, length $=20 \mathrm{~mm}$, and free-fall height $=4$ $\mathrm{cm})$ to disintegrate the microcapsules on the fabric samples. Subsequently, the cap was quickly sealed. After equilibrating at $25{ }^{\circ} \mathrm{C}$ for $5 \mathrm{~min}$, a manual extraction head (SUPELCO, $75 \mu \mathrm{m}$ $\mathrm{CAR} / \mathrm{PDMS}$ ) that was already activated at $250{ }^{\circ} \mathrm{C}$ for $30 \mathrm{~min}$ was inserted into the vial for $10 \mathrm{~min}$ for absorption. The extraction head was subsequently plugged into the inlet of the gas chromatograph (for $1 \mathrm{~min}$ at $250{ }^{\circ} \mathrm{C}$ ).

GC-MS was performed using a $6890 \mathrm{~N}$ Network GC system with a 5975 inert mass-selective detector (Agilent Technologies Inc.) equipped with an HP-5 column $(60 \mathrm{~m} \times 0.25 \mathrm{~mm}, 0.25$ $\mu \mathrm{m})$. The carrier gas was high-purity helium that was flowed at a constant rate of $1 \mathrm{~mL} \mathrm{m^{-1 }}$ and a split ratio of $10: 1$. The oven temperature was maintained at $50{ }^{\circ} \mathrm{C}$ for $4.5 \mathrm{~min}$, heated to $260{ }^{\circ} \mathrm{C}$ at a rate of $3{ }^{\circ} \mathrm{C} \mathrm{min}{ }^{-1}$, heated to $270{ }^{\circ} \mathrm{C}$ at a rate of $10{ }^{\circ} \mathrm{C} \mathrm{min}{ }^{-1}$, and maintained at $270{ }^{\circ} \mathrm{C}$ for $10 \mathrm{~min}$. MS was operated in the electron impact ionization mode $(70 \mathrm{eV})$, and the interface, source, and quadrupole temperatures were set as $280{ }^{\circ} \mathrm{C}, 230{ }^{\circ} \mathrm{C}$, and $150{ }^{\circ} \mathrm{C}$, respectively. The mass range was $33-$ $320 \mathrm{~m} / \mathrm{z}$.

The resulting mass spectra were analyzed by manual spectral analysis based on the NIST11 library and a self-built library to identify the compounds. Further, the volatile compounds were quantified based on the comparison of the GC peak heights.

\section{Results and discussion}

\subsection{Microcapsule characterization}

3.1.1 FTIR analysis of microcapsules. The FTIR spectra of the essence oil (a), microcapsules containing essence oil (b), and the shell material MMF resin (c) were presented in Fig. 2. As is shown in curve a, the peaks at 752,1243, 1367 and $1733 \mathrm{~cm}^{-1}$ corresponded to the $\mathrm{C}=\mathrm{O}$ stretching vibration, $\mathrm{C}-\mathrm{H}$ deformation vibration, $\mathrm{C}-\mathrm{O}$ stretching vibration, and deformation vibration of ortho-substituted phenyl ring, respectively. In curve c, the peaks at 814 and $1558 \mathrm{~cm}^{-1}$ corresponded to the bending vibrations of triazine ring and they were the characteristic peaks of MF resins. ${ }^{23}$ The peak at $2358 \mathrm{~cm}^{-1}$ indicates unreacted $-\mathrm{CN}$ groups, whereas the strong and wide peak at approximately $3412 \mathrm{~cm}^{-1}$ indicated the superposition of $\mathrm{O}-\mathrm{H}$ and $\mathrm{N}-\mathrm{H}$ stretching vibrations. All these absorption peaks could be found

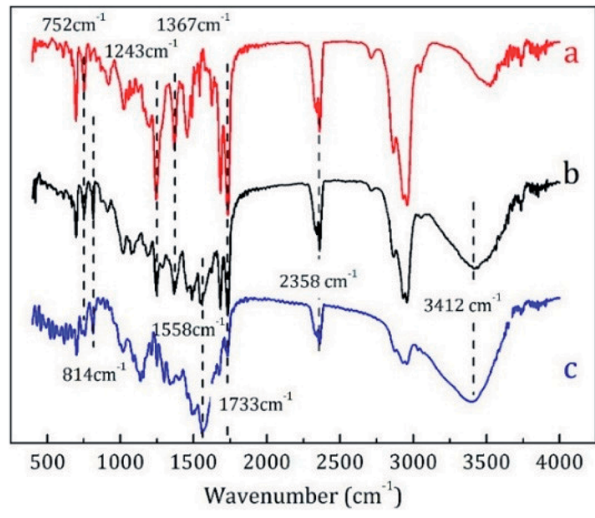

Fig. 2 The Fourier transform infrared spectra of the (a) essence oil, (b) microcapsules, and (c) MMF resin.

in curve b, demonstrating that the core material was successfully encapsulated by MMF resin.

3.1.2 Microcapsule structure and particle size. As mentioned in our previous works, ${ }^{15,22}$ we had tried to control the microencapsulation process based on the mechanism, and realized the particle size controlling of prepared microcapsules by change the system fluid flow conditions (such as agitation speed, agitator paddle type and reactor geometry). In this work, the micro-morphology and particles size of prepared microcapsule samples were also characterized, and the obtained data was used for conducting further studies. The microcapsule structure was observed via SEM, and it was found that the prepared microcapsules were observed to exhibit regular spherical shapes with smooth surfaces (Fig. 3a). Upon destruction, the inner surfaces of the microcapsules were smooth, and the cross section of the shell was uniform (Fig. 3b).

Table 2 presents the $D_{50}, P$, and $E \%$ values of the microcapsule samples. Microcapsule samples 1-5 exhibited different average particle sizes, narrow size distributions, and high coating efficiencies. The corresponding optical morphologies are denoted in Fig. $4 a_{1}-e_{1}$. The samples were relatively uniform throughout the large optical field.

\subsection{Fabric application efficiency and broken release characteristics of microcapsules}

3.2.1 Microscopic image analysis of microcapsules in textile functional finishing. Textile functional finishing is one of
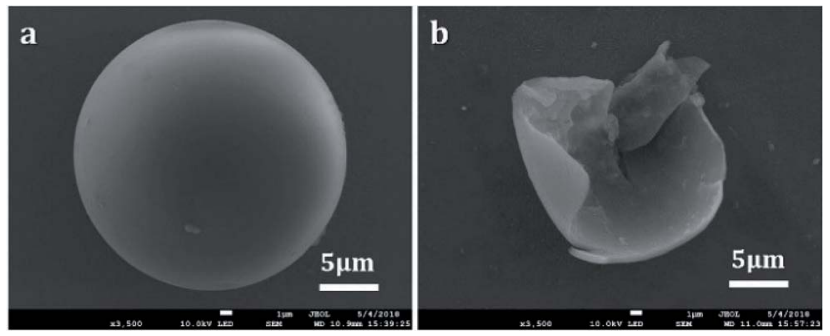

Fig. 3 SEM images denoting the morphology of the prepared microcapsules: (a) as-prepared microcapsule and (b) broken microcapsule. 
Table 2 Parameters of the prepared microcapsule samples

\begin{tabular}{lccl}
\hline Sample & $D_{50}(\mu \mathrm{m})$ & $P$ & $E \%$ \\
\hline a & 37.69 & 1.19 & $89.25 \%$ \\
b & 32.91 & 1.13 & $90.35 \%$ \\
c & 23.65 & 1.26 & $86.06 \%$ \\
d & 12.01 & 1.31 & $85.40 \%$ \\
e & 6.67 & 1.16 & $86.52 \%$ \\
\hline
\end{tabular}

the major applications of aromatic microcapsules. The microcapsules impregnated in a fabric will be gradually destroyed by external forces during use, leading to the exposure of the encapsulated core material causing explosive release effect and prolonged aroma release. This release performance is critical
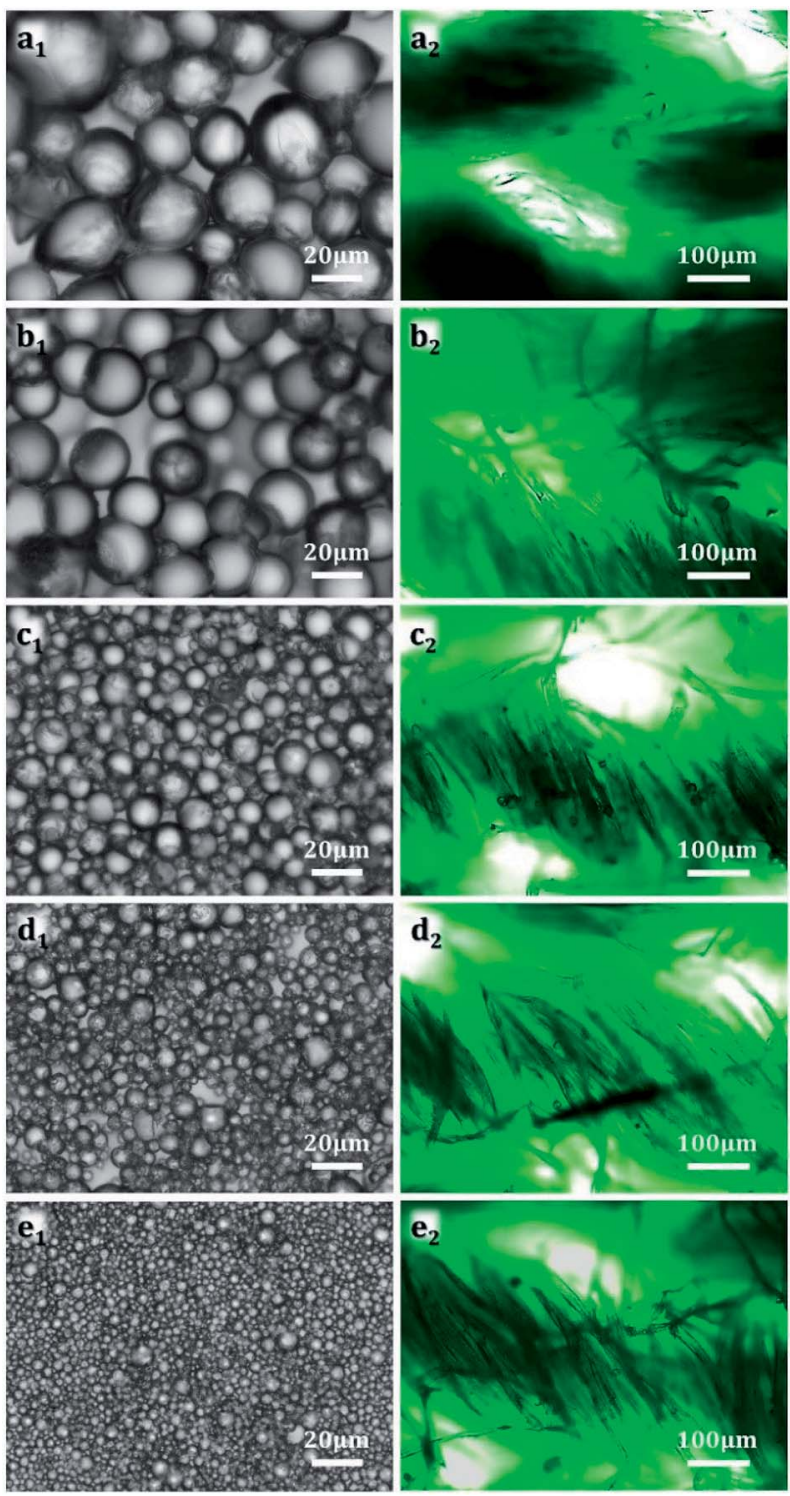

Fig. 4 OM images denoting the morphologies of prepared microcapsules having different particle sizes $\left(a_{1}-e_{1}\right)$ and the corresponding fabric samples finished by microcapsules $\left(a_{2}-e_{2}\right)$. while evaluating microcapsules. In this study, we treated fabrics with the prepared microcapsules without using additives (e.g., binders and penetrants). The OM images of the microcapsuletreated fabrics are depicted in Fig. $4 \mathrm{a}_{2}-\mathrm{e}_{2}$.

The microcapsule size affected the positions of the microcapsules on the fabrics, which will affect the impregnation efficiency. The large microcapsules were inserted at the intersections of fiber bundles (threads), as depicted in Fig. $4 \mathrm{a}_{2}$ and $b_{2}$. In case of the small microcapsules, the microcapsules tended to be adsorbed and stably fixed between individual fibers because of dimension matching. The availability of suitable spaces for microcapsule attachment increased with decreasing particle size, causing an increase in the impregnation efficiency (Fig. $4 \mathrm{c}_{2}$ ). When the particle size of microcapsules became smaller than the fiber diameter (Fig. $4 \mathrm{~d}_{2}$ and $\mathrm{e}_{2}$ ), it was difficult for the microcapsules to remain between the threads or fibers, and microcapsules were more likely to adhere to the surfaces of single fibers, decreasing the impregnation efficiency.

3.2.2 Broken release characteristics of microcapsules in textile functional finishing. The microcapsule impregnation efficiency was preliminarily evaluated via microscopic image analysis; however, the broken release intensity could not be accurately determined based on the images. Therefore, SPMEGC-MS was applied to study the broken release characteristics and impregnation efficiency. The samples were first treated using the hitting process described in Section 2.4.6, which simulated the external forces that the microcapsule-finished fabrics were subjected to during actual use.

Fig. 5 denotes the chromatographic elution curve of the fabric treated using microcapsule sample 1 . The following peaks are marked in the curve: A, ethyl-2-methylbutyrate; $\mathrm{B}$, ethyl 2-methylvalerate; C, 1,8-cineole; D, dihydromyrcenol; E, tetrahydrolinalool; F, isobornyl acetate; G, 4-tert-butylcyclohexyl acetate; H, 3,5,5-trimethylhexyl acetate; I, tricyclodecenyl propionate; J, isoamyl salicylate; and $\mathrm{K}$, $\alpha$-hexylcinnamaldehyde. The elution curve was similar to that of the microcapsules prepared in a non-ionic system in our previous study. ${ }^{24}$ The results indicated that the core material could be finely encapsulated and highly restored the release characteristics.

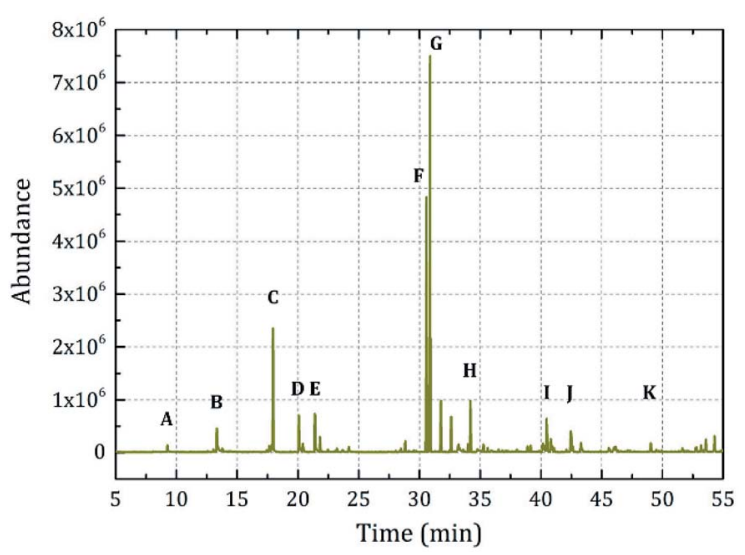

Fig. 5 SPME-GC-MS elution curve of the fabrics treated using microcapsule sample 1. 
To minimize the error and reduce the peak interference, 1,8eucalyptol was selected as the characteristic substance in the core material based on the suggestion of perfumers. The mass spectrum of 1,8-eucalyptol was depicted in Fig. 6I.

The SPME-GC-MS elution curves of the fabrics treated using the microcapsule samples 1-5 are depicted in Fig. 6II. The elution time of 1, 8-eucalyptol was approximately $17.9 \mathrm{~min}$ under the selected conditions. Because the selected characteristic peak often overlaps with the peak of D-limonene (the smaller one on its left side), it was difficult to express the intensity of 1,8-eucalyptin by peak area. Thus, the peak height was selected as the semi-quantitative factor.

The curves a, b, and c in Fig. 6II, which correspond to the fabrics finished by microcapsules with $D_{50}=37.69,32.91$, and $23.65 \mu \mathrm{m}$, respectively, showed strong peak intensity. The peak intensity of curve d $\left(D_{50}=12.01 \mu \mathrm{m}\right)$ was low, whereas curve e $\left(D_{50}=6.67 \mu \mathrm{m}\right)$ showed little scent characteristic. Because the $E \%$ values of different samples were similar, no significant difference could be observed between the core material loadings of the samples. Thus, the microcapsule impregnation efficiency was the major factor affecting the flavor release characteristics. No additives were used in this study to enhance the contact and adhesion between the microcapsules and the fabric substrate during impregnation; therefore, the microcapsules were mainly embedded and adsorbed onto the fabric. Based on Fig. 7, which denoted the peak height as a trend line of the particle size, and the discussion in Section 3.2.1, it could be conclude that the impregnation efficiency was dependent on the microcapsule particle size. As depicted in Fig. 7, the microcapsule particle size corresponding to the maximum peak height was approximately $25-30 \mu \mathrm{m}$. The diameter of a single fiber in the fabric substrate was also approximately 25-30 $\mu \mathrm{m}$, implying the maximization of the impregnation efficiency when the dimensions of the fibers and microcapsules were similar. This result could complement the phenomenon obtained by human sniffing (the human sniffing can clearly identify a 6.67 $\mu \mathrm{m}$ microcapsule-finished fabric sample with a light flavor odor even though it is difficult to distinguish between the odor intensities of samples $b$ and $c$ ).

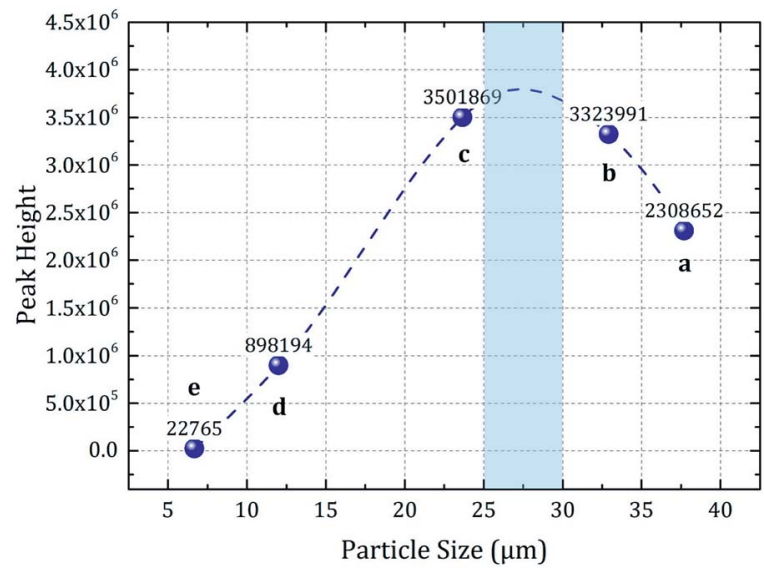

Fig. 7 Relation between the peak height of the SPME-GC-MS characteristic peak and the average microcapsule particle size.

All these results indicate that the selection of a suitable microcapsule size can improve the microcapsule performances in textile functional finishing. When the microcapsule and fiber sizes are similar, the microcapsules will easily and strongly adsorb onto the fabric, even in the absence of adhesives. Removing the need for binders in textile functional finishing will be helpful to improve the odor/function refreshing and feel of the textile.

\subsection{Sustained release behaviours of microcapsules having different particle sizes}

Sustained release is another important property of microcapsules. The sustained release of microcapsules in air is based on the permeation and migration of core material into shell along with its diffusion on shell surface. Therefore, the microcapsule particle size significantly influences the sustained release characteristics.

The prepared microcapsules having different particle sizes were placed in an air-circulating oven to simulate the dynamic release conditions of actual application. After $2400 \mathrm{~h}$, the

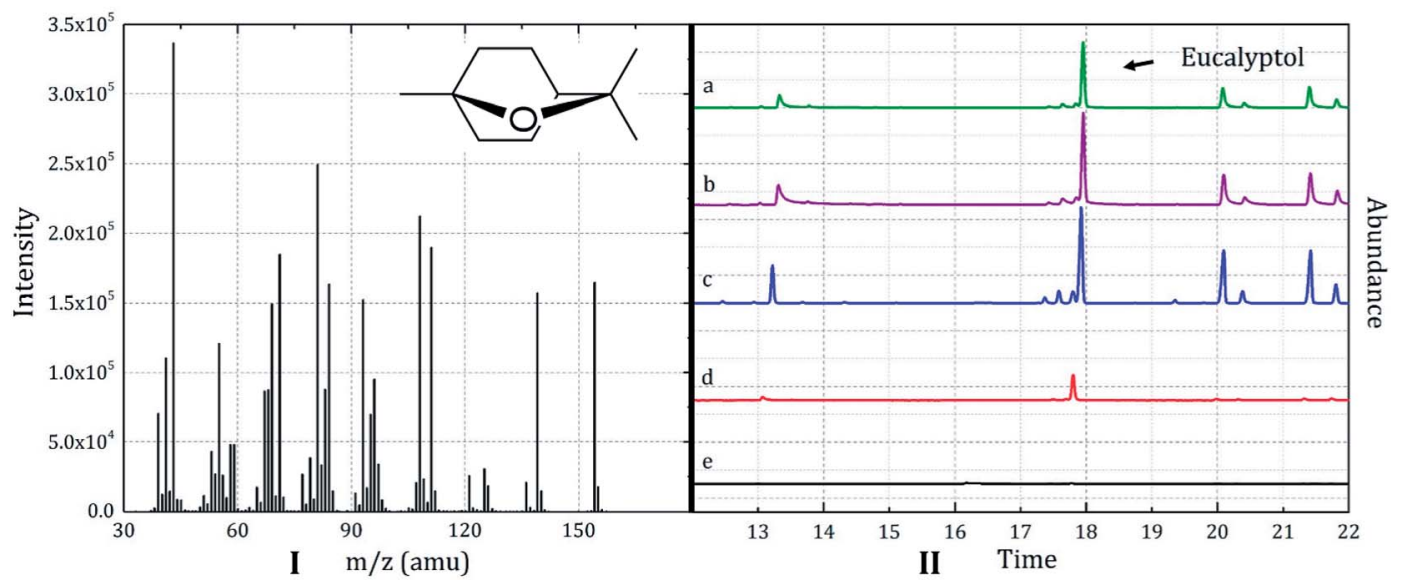

Fig. 6 (I) Mass spectra of 1,8-eucalyptol in the core material and (II) SPME-GC-MS elution curves of the fabrics treated by (a) sample 1, (b) sample 2, (c) sample 3, (d) sample 4, and (e) sample 5. 
release rate (rate of core material mass loss) was obtained (Fig. 8). The release occurred slowly, and the rate continuously decreased with time. Meanwhile, it significantly increased as the particle size decreased (curves a-e). To further analyze the variations in release rate, we fitted the sustained release data with different models: Higuchi equation, first dynamic equation and Peppas equation. However, in the actual fitting stage, the fitting results of Higuchi equation and first kinetic equation showed a certain deviation from the experimental results. And it was found that the Peppas model was more suitable for sustained release data fitting analysis. Additionally, in our experimental section, the internal air of air-circulating oven (Fig. 1 in 2.4.5) was connected to the atmosphere (an infinitely diluted system), and the equilibrium amount of core material released could be considered to almost 0 , which made the traditional equation physically unreasonable. Thus, a deformed Peppas equation was used as shown in eqn (6), and the fitting results were denoted by the dashed lines in Fig. 8 and Table 3 .

$$
y=100-a t^{k}
$$

In which, the release rate $(y)$ of microcapsules was the change of remaining essence oil percentages during sustained release time $(t)$.

According to the Adj. $R^{2}$ values, the Peppas model provided a good fit with the original data trend and can be used to predict the sustained release characteristics of the microcapsules. Value $k$ is the diffusional exponent related to the type of release behaviors and mechanisms as Fickian diffusion $(k=0.5)$, anomalous diffusion $(0.5<k<1.0)$, or non-Fickian diffusion $(k$ $=1.0) \cdot{ }^{18,25-28}$ In this study, the $k$ values of samples a and c-e were less than 0.5 , which illustrated that their release mechanisms were Fickian. This was due to the melamine resin microcapsule shell belonged to an insoluble and infusible three-dimensional cross-linked polymer, and the release environment was also an infinitely diluted system. The $k$ value of sample b was 0.5286 , which belonged to the non-Fickian diffusion from the mathematical point of view, but we believed that this was caused by

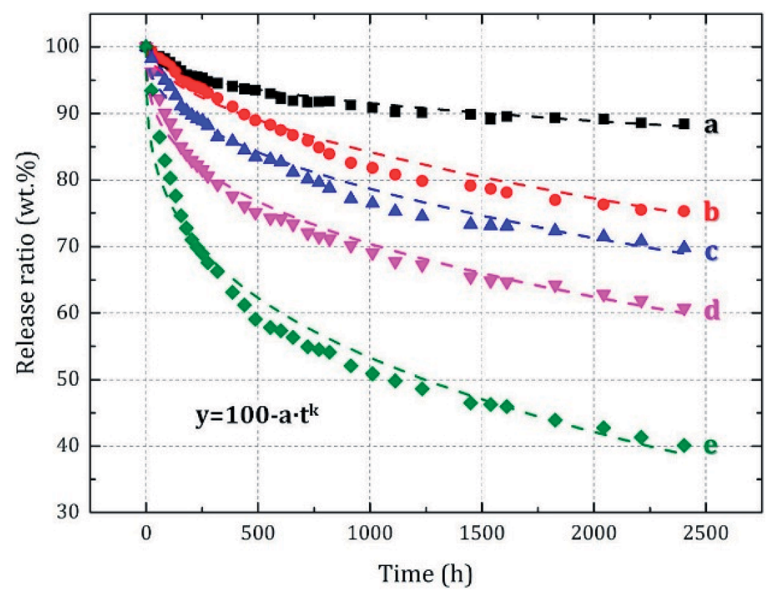

Fig. 8 The sustained release data and fitting curves for microcapsules having different particle sizes.
Table 3 Sustained release curve-fitting results for microcapsules with different particle sizes

\begin{tabular}{llll}
\hline Sample & $a$ & $k$ & Adj. $R^{2}$ \\
\hline a & 0.5367 & 0.3986 & 0.9704 \\
b & 0.4098 & 0.5286 & 0.9836 \\
c & 1.1079 & 0.4281 & 0.9803 \\
d & 2.7217 & 0.3453 & 0.9837 \\
e & 5.5378 & 0.3087 & 0.9760 \\
\hline
\end{tabular}

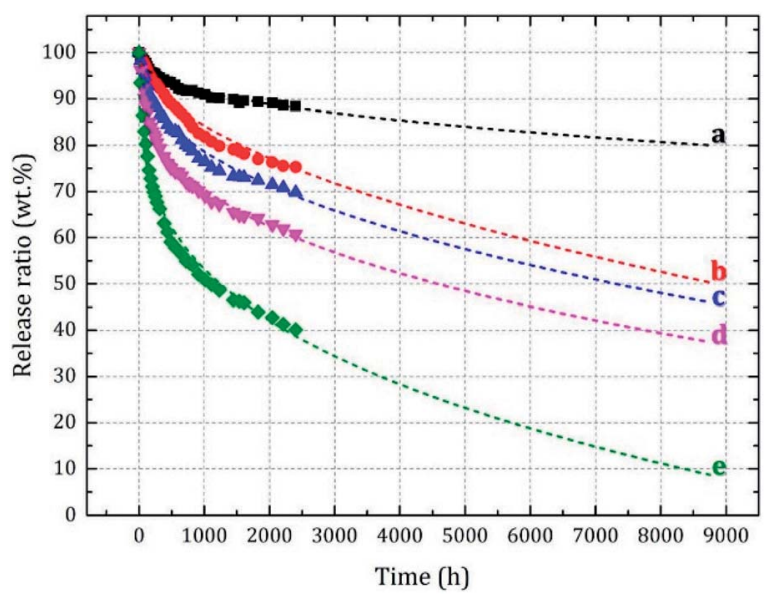

Fig. 9 Predicted sustained release characteristics of microcapsules having different particle sizes.

the test and fitting error or deviation, ${ }^{29-31}$ and it should actually be the Fickian diffusion mechanism too.

The predicted release characteristics over a period of one year are denoted in Fig. 9. The core material of the microcapsule sample having a small particle size $\left(D_{50}=6.67 \mu \mathrm{m}\right)$ was predicted to be substantially depleted after one year of sustained release, whereas the sample with a large particle size $\left(D_{50}=\right.$ $37.69 \mu \mathrm{m}$ ) released only approximately $10 \%$ of the core material. Thus, the large microcapsules showed better leak tightness and the small microcapsules exhibited a faster sustained release rate, indicating the microcapsules with different particle sizes having different application potentials.

\section{Conclusions}

In this study, the prepared MMF shell aromatic microcapsules were applied in textile functional finishing. After basic characterization, the relation between the microcapsule size and impregnation efficiency was evaluated by microscopic image analysis. And the SPME-GC-MS analysis indicated that the fabrics finished using microcapsules having an average particle size of 25-30 $\mu \mathrm{m}$ exhibited the optimal broken release performance among the synthesized microcapsules. The results denoted that the selection of a suitable microcapsule size can improve microcapsule performances in textile functional finishing. When the microcapsule size and fabric fiber diameter 
were similar, the highest impregnation efficiency and broken release intensity could be obtained.

The relation between the microcapsule size and sustained release behavior was also analyzed based on the sustained release curves. The sustained release data were observed to fit well with the Peppas model. Further, the release of core material from microcapsules was slow, and the release rate decreased with time. Particularly, the release rate increased as the microcapsule size decreased. Thus, the large microcapsules showed better leak tightness, whereas the small microcapsules exhibited a faster sustained release rate.

We believe that controlling the release characteristics of microcapsules by adjusting the particle size is a simpler, more adaptable and targeted strategy.

\section{Conflicts of interest}

There are no conflicts to declare.

\section{Acknowledgements}

The present study was financially supported by the National Natural Science Foundation of China, grant numbers 51703155, 51578360, 51778398, 51703154.

\section{References}

1 M. A. Teixeira, O. Rodríguez, S. Rodrigues, I. Martins and A. E. Rodrigues, AIChE J., 2012, 58, 1939-1950.

2 A. Sansukcharearnpon, S. Wanichwecharungruang, N. Leepipatpaiboon, T. Kerdcharoen and S. Arayachukeat, Int. J. Pharm., 2010, 391, 267-273.

3 S. Ghayempour and M. Montazer, J. Microencapsulation, 2016, 33, 497-510.

4 K. Son, D. I. Yoo and Y. Shin, Chem. Eng. J., 2014, 239, 284289.

5 C. Wang, W. Ye, Y. Zheng, X. Liu and Z. Tong, Int. J. Pharm., 2007, 338, 165-173.

6 C. Lin, Y. Li, M. Yu, P. Yang and J. Lin, Adv. Funct. Mater., 2007, 17, 1459-1465.

7 S. Fu, C. Xu, C. Du, A. Tian and M. Zhang, Colloids Surf., A, 2011, 384, 68-74.

8 P. Sánchez, M. V. Sánchez-Fernandez, A. Romero, J. F. Rodríguez and L. Sánchez-Silva, Thermochim. Acta, 2010, 498, 16-21.

9 T. Khadiran, M. Z. Hussein, Z. Zainal and R. Rusli, Sol. Energy Mater. Sol. Cells, 2015, 143, 78-98.
10 S. R. White, N. R. Sottos, P. H. Geubelle, J. S. Moore, M. R. Kessler, S. R. Sriram, E. N. Brown and S. Viswanathan, Nature, 2001, 409, 794-797.

11 D. Sun, G. Sun, X. Zhu, A. Guarin, B. Li, Z. Dai and J. Ling, Adv. Colloid Interface Sci., 2018, 256, 65-93.

12 N. Wang, Y. Wu, L. Mi, J. Zhang, X. Li and Q. Fang, J. Therm. Anal. Calorim., 2014, 118, 349-357.

13 A. Lavrenova, J. Farkas, C. Weder and Y. C. Simon, ACS Appl. Mater. Interfaces, 2015, 7, 21828-21834.

14 Y. Ma, Z. Li, H. Wang and H. Li, J. Colloid Interface Sci., 2019, 534, 469-479.

15 X. Fei, H. Zhao, B. Zhang, L. Cao, M. Yu, J. Zhou and L. Yu, Colloids Surf., A, 2015, 469, 300-306.

16 R. Mercadé-Prieto, X. Pan, A. Fernández-González, Z. Zhang and S. Bakalis, Ind. Eng. Chem. Res., 2012, 51, 16741-16749.

17 B. Peña, C. Panisello, G. Aresté, R. Garcia-Valls and T. Gumí, Chem. Eng. J., 2012, 179, 394-403.

18 F. M. Bezerra, O. G. Carmona, C. G. Carmona, M. J. Lis and F. F. de Moraes, Cellulose, 2016, 23, 1459-1470.

19 K.-W. Yeh, C. P. Chang, T. Yamamoto and T. Dobashi, Colloids Surf., A, 2011, 380, 152-155.

20 K. W. Yeh, C. P. Chang, T. Yamamoto and T. Dobashi, Colloid Polym. Sci., 2012, 290, 1583-1591.

21 R. Mercade-Prieto, R. Allen, D. York, J. A. Preece, T. E. Goodwin and Z. Zhang, J. Microencapsulation, 2012, 29, 463-474.

22 H. Zhao, X. Fei, B. Zhang, S. Zhao, G. Li and L. Cao, Particuology, 2019, 43, 38-45.

23 F. Salaün and I. Vroman, Eur. Polym. J., 2008, 44, 849-860.

24 H. Zhao, X. Fei, L. Cao, B. Zhang and X. Liu, Materials, 2019, 12(3), 393.

25 A. Ebadi, A. A. Rafati, S. Bavafa and M. Mohammadi, Appl. Surf. Sci., 2017, 425, 282-290.

26 S. Dash, P. N. Murthy, L. Nath and P. Chowdhury, Acta Pol. Pharm., 2010, 67, 217-223.

27 P. Costa, J. Manuel and S. Lobo, Eur. J. Pharm. Sci., 2001, 13, 123-133.

28 S. Cortés-Camargo, P. E. Acuña-Avila, M. E. RodríguezHuezo, A. Román-Guerrero, V. Varela-Guerrero and C. Pérez-Alonso, Food Res. Int., 2019, 116, 1010-1019.

29 J. M. Sankalia, M. G. Sankalia and R. C. Mashru, J. Controlled Release, 2008, 129, 49-58.

30 S. Asnaashari, N. S. Khoei, M. H. Zarrintan, K. Adibkia and Y. Javadzadeh, Pharm. Dev. Technol., 2011, 16, 400-407.

31 T. R. J. Heathman, W. R. Webb, J. Han, Z. Dan, G. Q. Chen, N. R. Forsyth, A. J. El Haj, Z. R. Zhang and X. Sun, J. Pharm. Sci., 2014, 103, 2498-2508. 